\title{
Cisplatin-resistant lung cancer cells maintain their high-migration ability by strong autophagy activity
}

\author{
Fenmiao Zhong, Ruiqi Wang, Zhiming Zhang
}

Nanjing Foreign Language School, China

Keywords: Non-small cell lung cancer, Migration, Lung cancer resistance, Autophagy.

\begin{abstract}
Lung cancer is one of the most prevalent malignancies worldwide. It is the leading cause of cancer deaths in the world. Non-small cell lung carcinoma (NSCLC) is the most common type of lung cancer. (accounting for more than 85\% of total lung cancer). Drug resistance, a major obstacle to successful cancer chemotherapy, frequently occurs in recurrent or metastatic lung cancer and results in poor clinical response. Clinically, with lung cancer patients develop drug resistance, the tissue of their primary lesions shows a stronger ability to invade and metastasize. Autophagy is a conservative catabolic process that acts as a "housekeeper" in eliminating protein aggregates and in organelles. Autophagy is closely related to cancer tumorigenesis. However, it is still unclear whether autophagy plays a role in lung cancer resistance. The subject of the experiment was studied by using a non-small cell lung cancer cell line A549 and its cisplatin-resistant cell line A549 DDP. The cell migration assay was used to detect the function of tumor cells, and autophagy flux was observed by immunoblotting. After blocking with the autophagy inhibitor Chloroquine, the migration ability of the tumor cells was examined. The results showed that A549 DDP resistance to cisplatin was significantly improved compared with A549, and migration ability of A549 DDP was stronger than that of A549, and the level of autophagy was also enhanced significantly. After autophagy was blocked by Chloroquine, the migration ability was reduced significantly. These indicate that an increase in autophagy activity contributes to maintaining the migration of drug-resistant cell lines.
\end{abstract}

\section{Introduction}

As one of the most common malignant tumors, lung cancer is characterized by high morbidity and mortality rates. In recent two years, the mortality and morbidity of lung cancer have gradually increased, which seriously threatens the lives and health of people all over the world. Non-small cell lung carcinoma (NSCLC) is the most common type of lung cancer, accounting for about $85 \%$ of the total number of lung cancer. Current main treatments for lung cancer include surgery, chemotherapy, radiotherapy, and targeted therapy. However, the therapeutic effect of lung cancer patients is still unsatisfactory, the most important reason counting for its failure is the resistance of lung cancer cells to treatment. At present, the treatment of advanced NSCLC is chemotherapy combined with cisplatin (DDP). However, cisplatin tolerance is the main cause of chemotherapy failure in some patients [2, 3, 4].

Autophagy includes three types: microautophagy, chaperone-mediated autophagy, and macrophagy. Autophagy is a tightly regulated strategy developed by cells to provide energy and nutrients by digesting long-lived or damaged cytosolic proteins and organelles. (such as protein polymers, organelles, etc.). The activation of ULK1 complexes (ULK1, ULK2, ATG13, FIP200, and ATG1101) activates class III PI3 K complexes (VPS34, ATG14, UVRAG and AMBRA 1), all of which can be activated and promoted by Beclin1, that initiates autophagy. These consist of the formation of the Atg5/Atg12/Atg16 complex; or the conjugation of LC3 to the phosphatidyl-etanolamine (PE), thus forming LC3-II. Then the autophagy membrane was extended. Therefore LC3-II is usually used as autophagy marker [5]. Many studies have shown that autophagy is involved in the occurrence, development, and metastasis of cancer. However, with the change of the environment in which cells live [6], autophagy has different functions at the early and advanced 
tumor. Autophagy suppress tumor initiation through reducing oxidative stress and DNA damage by inhibiting the accumulation of dysfunctional mitochondria. [7]. Moreover, once the tumor is formed, autophagy can promote its progress in various ways. Besides, autophagy can also promote malignant progression of tumors by reducing metabolic stress and promoting proliferation, migration, and invasion of highly metastatic tumors [7, 8, 9, 10, 11].

The primary tissue showed stronger invasive and metastatic ability after cisplatin resistance in advanced NSCLC, which was the main reason for poor prognosis in advanced NSCLC patients. In view of the existing report, autophagy promotes the proliferation, migration, and invasion of highly metastatic cancer cells, thus playing a role in promoting malignant progression of cancer. It is still unclear whether the resistance of advanced NSCLC to cisplatin and whether the enhanced migration ability in resisted cells is related to autophagy. This study explored this issue. The results showed that non-small cell lung cancer cells showed significantly enhanced migration ability and increased autophagy levels after resistance to cisplatin. Further experiments showed that A549/DDP maintains their migration ability with high levels of autophagy.

\section{Experimental methods and materials}

\subsection{Materials}

Human lung cancer A549 and cisplatin-resistant A549/DDP cell lines constructed in our laboratory were used; RPMI1640 complete culture medium was purchased from Gibco Company; Western Blo kit was purchased from Biyuntian Company; Rabbit anti-Human Monoclonal Antibody LC3 and Mouse anti-Human Monoclonal Antibody actin were purchased from Abcam and Sigma Company respectively. Transwell reagent and six-well plates were purchased from Corning Company; MTS reagent was purchased from Millipore Company. Baf A1 and CQ reagents were purchased from Abcam and Sigma respectively. Buy streptomycin and penicillin from Gibco. The other consumables used in this experiment were purchased from Corning Company.

\subsection{Method}

\subsubsection{Cell Culture of Lung Cancer}

The corresponding cells were cultured in 10\% FBS, $100 \mathrm{U} / \mathrm{mL}$ penicillin and streptomycin RPMI 1640. Additional addition of cisplatin (DDP) to A549/DDP cell culture medium was required, and the final concentration of DDP was 2 ugM to maintain its drug-resistant phenotype.

\subsubsection{MTS experiment}

In each group, 2000 cells were suspended in $100 \mathrm{mu} \mathrm{L}$ complete medium containing different concentration gradients of cisplatin, then added to 96 well plates, and three blank control groups were set up at the same time. After 48 hours, the MTS reagent was added and incubated for 4 hours. The absorbance value was measured by the enzyme label at $490 \mathrm{nM}$. Analysis of data and mapping. The activity was $100 \%$ in the group without cisplatin.

\subsubsection{Transwell experiment}

For migration assay, A549 or A549/DDP cells and serum-free medium were inoculated into $8 \mathrm{~mm}$ pore cell culture inserts. Subsequently, the serum-containing medium was added outside the insert (i.e. in the outdoor), and the cells were placed in the incubator for 16 hours. Cells were immobilized in $4 \%$ polyformaldehyde (PFA) and then scraped from the inside of the insert to observe and photograph them under an inverted microscope. Repeat three times.

\subsubsection{Cell Scratch Test}

According to $3.0 \times 10^{5}$ holes per hole, lung cancer cells of each group were plated on the 6-well plate. When the cell growth density reached 90-95\%, the lines were drawn with the head of the gun against the ruler. After washing with PBS, the cells were replaced with serum-free medium and 
photographed. Subsequently, according to the instructions of each group, after adding CQ or not, they continued to cultivate for 48 hours and took pictures at the same shooting point.

\subsubsection{Western Blot experiment}

The well-growing cells were harvested and the lysate was cleaved. The protein liquid was collected and the protein concentration was measured. Constant voltage electrophoresis, membrane-constant current $276 \mathrm{~mA}$, wet rotation 120 minutes. After that, it was closed for 2 hours, added one antibody, shaking overnight at $4 \mathrm{C}$, incubated at room temperature for 1 hour with HRP labeled the second antibody, and exposed the PVDF film to the exposure machine after dropping ECL luminescent solution.

\subsubsection{Immunofluorescence}

Cell climbing tablets were placed in a 24-well plate. 30,000 cells from each group were plated in a 24-well plate. The cells were cultured overnight in complete medium. Baf A1 reagent was added 6 hours before treatment. The culture medium was removed and PBS was cleaned. Adding methanol for 15 minutes, after fixing, Tritone penetrated the membrane for 10 minutes, goat serum was blocked and then added the corresponding the first antibody. After washing first antibody, the fluorescent second antibody was added. After washing the second antibody, DAPI was added, sealed and photographed.

\subsection{Statistical Processing}

Data were analyzed with GraphPad Prism. The non-paired Student $t$ test was used to compare two groups. When $\mathrm{P}<0.05$, the difference was statistically significant.

\section{Experimental results}

\subsection{The cell viability of drug-resistant cell lines was compared with A549 cell lines at different concentrations of cisplatin.}

In this study, the IC50 method was used to detect the half inhibitory concentration of cisplatin. A549 cells and A549/DDP cells were treated with cisplatin at concentrations of 10, 20, 40 and $80 \mathrm{mu}$ M, respectively. The resistance of A549/DDP and A549 cells to cisplatin was verified. The results of Fig. 1 showed that with the increasing concentration of cisplatin, the viability of both groups decreased, but it is noteworthy that the decline of A549 DDP was more slowly. These results suggest that the model of cisplatin resistance in lung cancer cells was successfully simulated by A549/DDP constructed earlier in this experiment.

\begin{tabular}{|r|r|r|r|r|r|r|r|}
\hline A549 48h & & & & & \\
\hline & & 1 & 2 & 3 & 4 & 5 & 6 \\
\hline A & 1.6109 & 0.8165 & 0.4398 & 0.3044 & 0.1668 \\
\hline B & 1.52 & 0.8227 & 0.4467 & 0.3167 & 0.1972 \\
\hline C & 1.6054 & 0.8099 & 0.4517 & 0.3114 & 0.1883 \\
\hline & & & & & & \\
\hline A549/DDP & $48 h$ & & & & & & \\
\hline & & 1 & 2 & 3 & 4 & 5 & 6.593 \\
\hline A & & 1.5809 & 1.2268 & 0.8876 & 0.7067 & 0.593 \\
\hline B & & 1.5915 & 1.2937 & 0.8714 & 0.7119 & 0.5571 \\
\hline C & 1.6225 & 1.3154 & 0.8991 & 0.7099 & 0.5881 \\
\hline
\end{tabular}

Fig 1.A

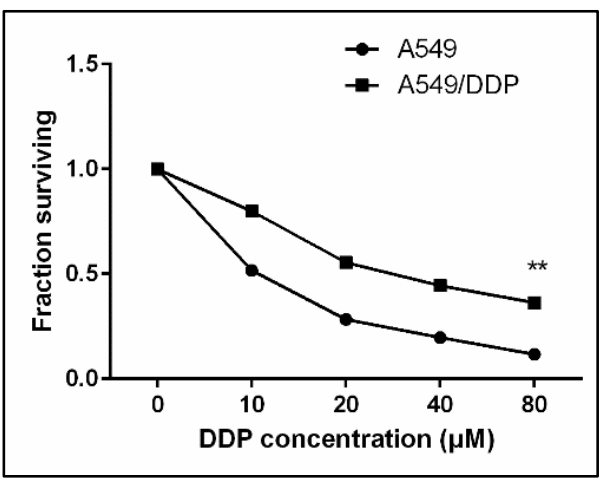

Fig2.B

Fig 1. Cell viability test at different concentrations of cisplatin

A549 cells and A549/DDP cells were treated with cisplatin at concentrations of 10, 20, 40 and 80 mu M, respectively. The plates were laid on 96-well plates. After 48 hours, OD values were 
measured by the MTS method, such as tables (A, B, C in the tables represent the data of three complex holes). B Drawing with Graphpad Prism software. (** $\mathrm{P}<0.01 ; \mathrm{n}=3$ )

\subsection{Detection of migration ability of lung cancer cell A549 compared with A549/DDP}

We performed a classical migration assay and scratch assay to study whether drug resistance can influence migration of A549 cells. . As shown in Figure 2A, the migration ability of A549 / DDP was significantly enhanced compared with A549 cell line. The scratch experiment in Fig. 2B was in line with that conclusion. The results showed that lung cancer cells had stronger migration ability after resistance to cisplatin.

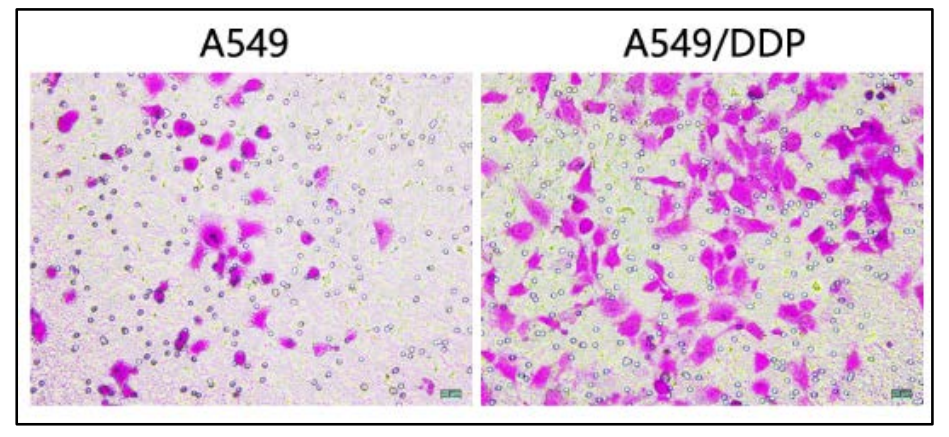

Fig 2.A

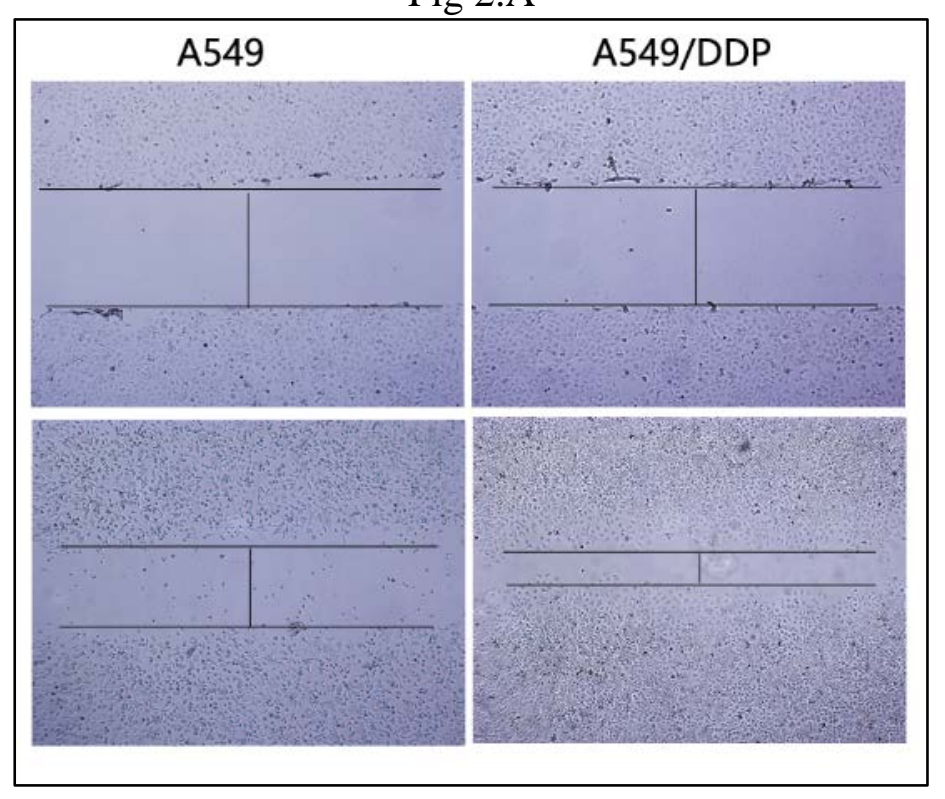

Fig2.B

Fig2.Cell transwell and Scratch Test

A549 and A549/DDP cells were cultured by the Transwell assay. $2 * 10^{4}$ cells were planted in a Transwell chamber. After 24 hours of culture, the cells were taken for examination and photograph. B. Cell scratch test, A549 and A549 / DDP cells were cultured respectively. $3 * 10^{5}$ cells were covered with six-well plates. After a scratch, PBS was washed and replaced with a serum-free medium. After 48 hours, the cells were taken out for photographs.

\subsection{Detection of Autophagy Level of A549/DDP in Lung Cancer Cells}

Previous studies have shown that autophagy can promote the proliferation, migration, and invasion of highly metastatic tumor cells and promote the malignant progression of tumors after tumorigenesis. Given the above conclusion, the migration level of lung cancer cells increased significantly after resistance to cisplatin. We assume that whether autophagy activity will change after cisplatin resistance in lung cancer cells To this end, we first compared the autophagy levels in drug-resistant cell lines and A549 cell lines. Results As shown in lanes 1 and 3 of Fig. 3A, the LC3-I 
level in A549 DDP did not change much, but the production of LC3-II, an autophagic marker, increased significantly. To further clarify whether the increase of LC3-II is due to the increase of early synthesis of autophagy or the blockage of degradation of late autophagy and lysosomes, we used the late inhibitor Baf A1 of autophagic flow. The results showed that LC3-II increased further when lung cancer cells were resistant to cisplatinas shown in lanes 2 and 4 of Figure 3A. The results showed that the synthesis of early autophagosome increased and the level of autophagy increased in A549/DDP. To show the change of autophagy activity more intuitively, we made further immunofluorescence. As shown in Figure 3B, A549 DDP could be observed to increase the number of LC3-B-positive puncta without autophagy inhibitor Baf A1, to further distinguish LC3-B-positive puncta was from early autophagy or late autophagy. Under Baf A1, we can only see the autophagyosome. The results proved that cisplatin resistance can facilitate the formation of autophagosomes in lung cancer cells.

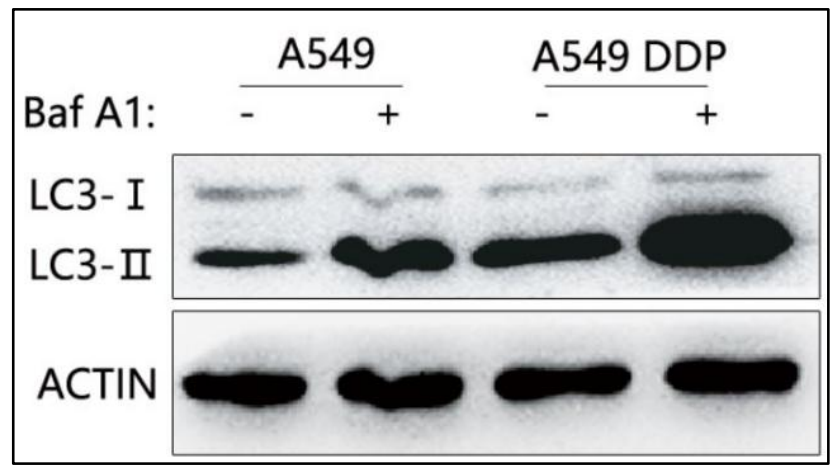

Fig3.A

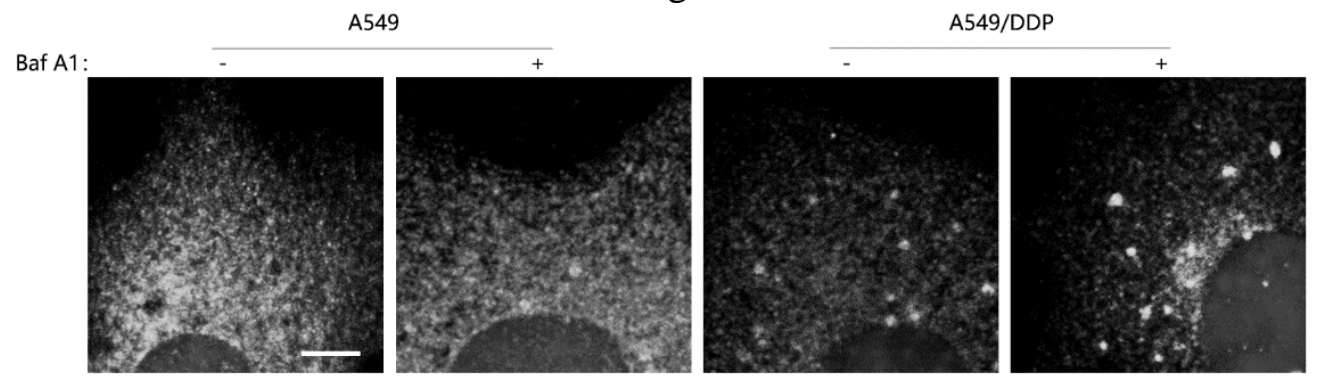

Fig3.B

Fig. 3 Expression of autophagy marker LC3 and observation of autophagosome

A549 cells and A549/DDP cells were cultured in groups with or without inhibitors, and protein was collected. Western Blot could track the expression of LC3. The synthesis of autophagosome was observed by immunofluorescent labeling LC3 before and after drug resistance in lung cancer cells. The inhibitors were added or not according to the instructions. Bar $=5 \mu \mathrm{m}$

\subsection{The increase of autophagy level is an important reason for the high migration ability of lung cancer drug-resistant cells.}

Considering that lung cancer cell lines resistant to cisplatin have stronger migration ability and a higher level of autophagy, we wonder whether the increase of autophagy level is an important reason for the strong migration ability of A549/DDP. To test this hypothesis, we used autophagy-lysosome inhibitor, Chloroquine (CQ), to suppress late autophagy-lysosome. Results as shown in Fig. 4A, the migration ability of A549/DDP decreased significantly after CQ treatment. The scratch test in Fig. $4 \mathrm{~B}$ also confirms the same conclusion. The results showed that the migration ability of A549/DDP depended on the increase of autophagy level. 


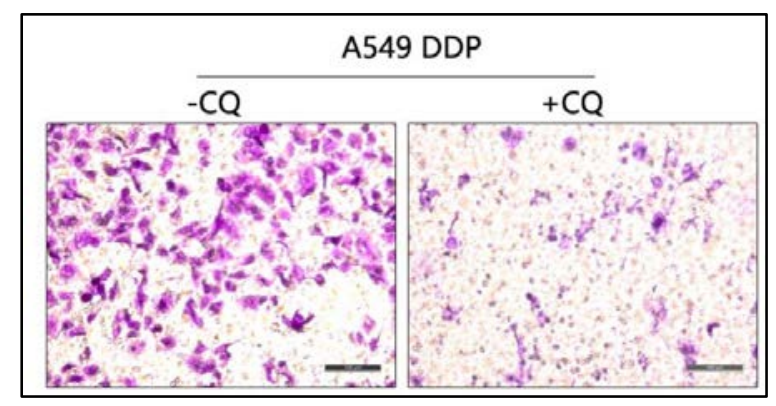

Fig 4. A

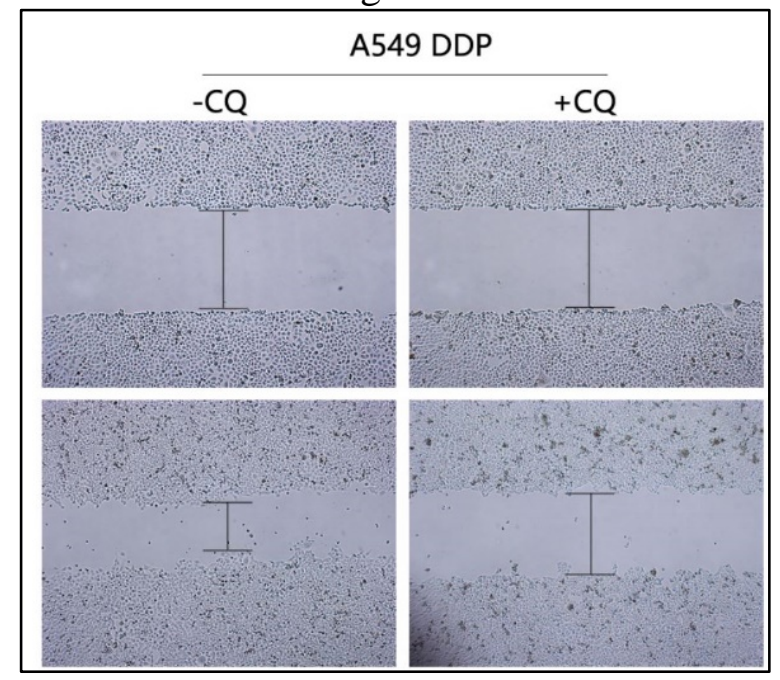

Fig 4. B

Fig4.Cell transwell and Scratch Test

A549/DDP cells were cultured by the Transwell assay. $2 * 104$ cells were collected and planted in Transwell chamber. CQ was added or not in groups. After 24 hours of culture, the cells were taken for examination and photograph. In the B cell scratch test, A549/DDP cells were taken and covered with 6-well plates. After a scratch, PBS was washed and replaced with a serum-free medium. After 48 hours, photos were taken.

\section{Discussion}

Lung cancer represents the leading cause of cancer-related mortality throughout the world. Among them, NSCLC represents a class of highly heterogeneous tumors [12]. There are many ways to treat NSCLC, including apoptosis-inducing chemotherapy drugs, such as cisplatin [12,13]. Unfortunately, chemotherapy resistance and disease recurrence remain major causes of lung cancer patient mortality, and recurrent tumors usually show stronger migration, invasion and metastasis ability and cisplatin-resistant characteristics [14-16]. This study demonstrated that chemotherapy-resistant cell lines have stronger migration ability than their parental cell line. It is suggested that the enhanced migration ability of lung cancer is one of the characteristics of cisplatin-resistant lung cancer.

Previous studies have shown that autophagy plays a role in tumor inhibition by reducing oxidative stress and DNA damage through inhibiting the accumulation of dysfunctional mitochondria in the early stage of tumor initiation. If Atg5 or Atg7 was deleted, the mice could develop liver tumors [9]. Beclin1 half-knockout mice can spontaneously form hepatocellular carcinoma [8]. Tumor suppressor genes such as PTEN and P53 can activate autophagy [10]. In fact, in the course of cancer progression, various environmental stress factors can enhance the autophagy activity of cells. For example, hypoxia, nutritional deprivation [22] and separation from ECM [23, 24], at the same time, these stress factors can promote tumor metastasis. For example, the increase of LC3B staining in 
immunohistochemistry is closely related to lymph node transformation and the decrease in the survival rate of human breast cancer [25, 26]. Tumor cells need adequate nutritional support, at which point the cancer cells will activate autophagy to produce energy, which is to cope with the need for supplements for their proliferation. Tumor cells can also activate autophagy to protect themselves from drug killing under the stimulation of external drugs. For example, tumor cells can degrade drug macromolecules through autophagy. Our results showed that the production of LC3-II, increased significantly when lung cancer cells were resistant to the cisplatin. LC3-II increased further with using BafA1, an advanced inhibitor of autophagic flow. The results showed that the autophagy level of lung cancer cells increased after resistance to cisplatin.

At present, It is generally accepted that autophagy plays a role in promoting tumors after the formation of tumors so that tumor cells develop in harsh environments [6,7]. Autophagy promotes invasion and migration of various types of cancer cells [7, 18, 19, 20, 21, 22, 23, 24]. For example, LC3B staining was also enhanced after melanoma metastasis [25, 26, 27, 28] compared with the original tumor samples. LC3B expression is also associated with the metastasis of hepatocellular carcinoma. Compared with primary tumors, LC3B expression is higher in metastatic tumors. Our experimental results showed that the migration ability of A549/DDP decreased significantly after CQ treatment. It indicated that high autophagy levels maintained high migration ability in A549/DDP.

To sum up, we simulated the drug resistance model of lung cancer in vivo and constructed a cisplatin-resistant A549 / DDP in the early stage. It was found that compared with the p arental cell lines, the migration ability and autophagy level of the drug-resistant strains were significantly improved. Further studies showed that CQ inhibited autophagy significantly reduced the migration ability of A549 / DDP cells. Our investigation indicated that high autophagy levels increased the migration ability of cisplatin-resistantcell lines, which had never been reported before. It is necessary to further study the specific molecular mechanism. It may be a new direction of anti-cancer research to take autophagy as a new target to overcome the relapse and drug resistance of NSCLC.

\section{References}

[1] Torre LA, Bray F, Siegel RL, Ferlay J, Lortet-Tieulent, Jemal A. Global cancer statistics, 2012. CA Cancer J Clin. 2015;65(2):87-108

[2] Yang M, Fan WF, Pu X, et al. Significance of thymidylate synthase expression for resistance to pemetrexedin pulmonary adenocarcinoma. Oncol Lett. 2014;7(1):227-232

[3] Johnson AM, Hines RB, Johnson JA, et al. Treatment and survival disparities in lung cancer: the effect of social environment and place of residence. Lung Cancer. 2014;83(3):401-407. doi: 10.1016/j.lungcan.2014.01.008

[4] Komaki R, Tsao AS, Mehran RJ. Non-small cell lung cancer 60 years of survival outcomes at The University of Texas MD Anderson Cancer Center. Springer New York, 2013: 45-62.

[5] Klionsky DJ. Autophagy: from phenomenology tomolecular understanding in less than a decade. Nat Rev Mol Cell Biol, 2007, 8: 931-7

[6] White E, Mehnert JM, Chan CS. Autophagy, metabolism, and cancer. Clin Cancer Res. 2015; 21:5037-46.

[7] Tian Y, Wang L, Ou J. Autophagy, a double-edged sword in hepatocarcinogenesis. Molecular \& Cellular Oncology. 2015; 2:e1004968.

[8] Guo JY, Karsli-Uzunbas G, Mathew R, Aisner SC, Kamphorst JJ, Strohecker AM, et al. Autophagy suppresses progression of K-ras-induced lung tumors to oncocytomas and maintains lipid homeostasis. Genes Dev. 2013; 27:1447-61.

[9] Mathew R, Karantza-Wadsworth V, White E. Assessing metabolic stress and autophagy status in epithelial tumors. Methods Enzymol. 2009; 453:53-81. 
[10] Rosenfeldt MT, O'Prey J, Morton JP, Nixon C, MacKay G, Mrowinska A, et al. p53 status determines the role of autophagy in pancreatic tumour development. Nature. 2013; 000:1-5.

[11] Sharifi MN, Mowers Erin E, Drake Lauren E, Collier C, Chen H, Zamora M, et al. Autophagy promotes focal adhesion disassembly and cell motility of metastatic tumor cells through the direct interaction of paxillin with LC3. Cell Rep. 2016; 15:1-13.

[12] Gridelli C, Rossi A, Carbone DP, Guarize J, Karachaliou N, Mok T et al. Non-small-cell lung cancer. Nat Rev Dis Primers 2015; 1: 15009.

[13] Schaake-Koning C, van den Bogaert W, Dalesio O, Festen J, Hoogenhout J, van Houtte P et al. Effects of concomitant Cisplatin and radiotherapy on inoperable non-small-cell lung cancer. N Engl J Med 1992; 326: 524-530.

[14] Olaussen KA, Dunant A, Fouret P, Brambilla E, Andre F, Haddad V et al. DNA repair by ERCC1 in non-small-cell lung cancer and Cisplatin-based adjuvant chemotherapy. N Engl J Med 2006; 355: 983-991.

[15] Mitsudomi T, Morita S, Yatabe Y, Negoro S, Okamoto I, Tsurutani J et al. Gefitinib versus Cisplatin plus docetaxel in patients with non-small-cell lung cancer harbouring mutations of the epidermal growth factor receptor (WJTOG3405): an open label, randomised phase 3 trial. Lancet Oncol 2010; 11: 121-128.

[16] Scagliotti GV, Parikh P, von Pawel J, Biesma B, Vansteenkiste J, Manegold C et al. Phase III study comparing Cisplatin plus gemcitabine with Cisplatin plus pemetrexed in chemotherapy-naive patients with advanced-stage non-small-cell lung cancer. J Clin Oncol 2008; 26: 3543-3551.

[17] Tian Y, Wang L, Ou J. Autophagy, a double-edged sword in hepatocarcinogenesis. Molecular \& Cellular Oncology. 2015; 2:e1004968

[18] Li J, Yang B, Zhou Q, Wu Y, Shang D, Guo Y, et al. Autophagy promotes hepatocellular carcinoma cell invasion through activation of epithelial mesenchymal transition. Carcinogenesis. 2013; 34:1343-51.

[19] Dikic I, Elazar Z. Mechanism and medical implications of mammalian autophagy. Nat Rev Mol Cell Biol. 2018; 349-364.

[20] Chaffer CL, Weinberg RA. A perspective on cancer cell metastasis. Science 2011; 331: 1559_ 1564.

[21] Valastyan S, Weinberg RA. Tumor metastasis: molecular insights and evolving paradigms. Cell 2011; 147: 275 -292.

[22] Kroemer G, Marino G, Levine B. Autophagy and the integrated stress response. Mol Cell 2010; 40: $280-293$.

[23] Avivar-Valderas A, Salas E, Bobrovnikova-Marjon E, Diehl JA, Nagi C, Debnath J et al. PERK integrates autophagy and oxidative stress responses to promote survival during extracellular matrix detachment. Mol Cell Biol 2011; 31: 3616-3629.

[24] Fung C, Lock R, Gao S, Salas E, Debnath J. Induction of autophagy during extracellular matrix detachment promotes cell survival. Mol Biol Cell 2008; 19: 797-806.

[25] Lazova R, Camp RL, Klump V, Siddiqui SF, Amaravadi RK, Pawelek JM. Punctate LC3B expression is a common feature of solid tumors and associated with proliferation, metastasis, and poor outcome. Clin Cancer Res 2012; 18: 370-379.

[26] Zhao H, Yang M, Zhao J, Wang J, Zhang Y, Zhang Q. High expression of LC3B is associated with progression and poor outcome in triple-negative breast cancer. Med Oncol 2013; 30: 475. 
[27] Lazova R, Klump V, Pawelek J. Autophagy in cutaneous malignant melanoma. J Cutan Pathol 2010; 37: 256-268.

[28] Han C, Sun B, Wang W, Cai W, Lou D, Sun Y et al. Overexpression of microtubuleassociated protein-1 light chain 3 is associated with melanoma metastasis and vasculogenic mimicry. Tohoku J Exp Med 2011; 223: 243-251. 